\title{
Delirium postoperatorio. Una ventana hacia una mejoría de la calidad y seguridad en la atención de pacientes quirúrgicos*
}

Drs. EDUARDO TOBAR A. ${ }^{1}$, MARIO ABEDRAPO M. ${ }^{2}$, JAIME GODOY C. ${ }^{3}$, CARLOS ROMERO P. ${ }^{1}$

1 Unidad de Cuidados Intensivos, Unidad de Pacientes Críticos, Departamento de Medicina.

2 Departamento de Cirugía.

3 Departamento de Anestesiología y Reanimación.

Hospital Clínico Universidad de Chile.

Santiago, Chile.

\begin{abstract}
Postoperative delirium. A window to quality improvement and safety in the care of surgical patients

Postoperative delirium or acute confusional state is a common complication among older subjects. Many factors influence its appearance, such as preexistent problems of the patient, medication use, pain, the perioperative anesthetic management and the intensity of the inflammatory reaction to surgical trauma. Its consequences are a longer hospital stay, higher risk of complications and a long term derangement of functional status and cognitive performance. The management of delirium is multifactorial, including the avoidance of precipitating factors, the maintenance of an adequate environment and the conscious use of neuroleptics. The prevention of delirium should be a priority that will improve health care standards.
\end{abstract}

Key words: Delirium, postoperative complications, acute confusional state, older people.

\section{Resumen}

El delirium postoperatorio constituye una complicación frecuente y relevante de los pacientes quirúrgicos, en particular en los adultos mayores. Su génesis es multifactorial participando características preexistentes del paciente, y gatillantes como medicamentos, dolor, el enfrentamiento anestésico peri operatorio y la intensidad de la respuesta inflamatoria asociada al trauma quirúrgico, entre otros. La aparición de delirium postoperatorio se asocia a desenlaces adversos, como una mayor estadía hospitalaria, mayor riesgo de complicaciones, y a una reducción en la funcionalidad y el estado cognitivo en la evolución alejada. Estrategias de prevención no farmacológicas multimodales, han documentado una reducción significativa en la incidencia de delirium. La terapia del delirium, debe enfocarse en la búsqueda y manejo de factores precipitantes, en favorecer un adecuado entorno no farmacológico, y en el uso apropiado de neurolépticos. El adecuado reconocimiento de esta entidad, y la implementación de estrategias de prevención no farmacológicas constituyen actualmente un estándar que promueve una atención de calidad y segura a los pacientes quirúrgicos.

Palabras clave: Delirium, complicaciones postoperatorias, envejecimiento.

*Recibido el 13 de enero de 2012 y aceptado para publicación el 12 de febrero de 2012.

Correspondencia: Dr. Eduardo Tobar A.

Santos Dumont 999, Santiago, Chile.

etobar@redclinicauchile.cl 


\section{Introducción}

El delirium corresponde a un trastorno de la función cerebral de inicio agudo, que pueden desarrollar los pacientes hospitalizados, en especial los adultos mayores. Se caracteriza por una alteración en el nivel de conciencia de curso fluctuante, y por alteraciones de grado variable en varios dominios del funcionamiento cerebral, tales como la organización del pensamiento, la relación con el entorno y, característicamente, un déficit en la atención. Ocasionalmente se manifiesta como agitación motora y síntomas mentales positivos (ilusiones, alucinaciones), pero lo más frecuente son las formas hipoactivas y la variante mixta.

Durante los últimos años, y luego de objetivarse una elevada incidencia de delirium, así como su asociación con desenlaces sanitarios adversos, esta condición se ha constituido en una oportunidad de mejoría de la atención hospitalaria a través de la implementación de estrategias de prevención multimodales.

El delirium postoperatorio (DPO) corresponde a aquel que aparece en pacientes que son sometidos a un procedimiento quirúrgico, y posee algunas características particulares que lo asemejan y/o diferencian del delirium en otras subpoblaciones de pacientes. El rol del acto quirúrgico y su respuesta inflamatoria posterior, el manejo anestésico peri operatorio, y el manejo del dolor postoperatorio, son algunas de las características particulares de este fenómeno en el paciente quirúrgico. La presente revisión aborda la importancia e impacto de esta condición, así como las estrategias de prevención y terapia recomendadas.

\section{Diagnóstico, epidemiología y factores de riesgo}

Para el diagnóstico de delirium tenemos la opción de recurrir a diversos instrumentos, según sea el escenario y la disponibilidad de psiquiatra. El estándar diagnóstico es la evaluación psiquiátrica, la cual en 20-30 minutos de entrevista al paciente, familiares y personal hospitalario, verifican la presencia de los criterios ICD-10 o DSM-IV para esta condición ${ }^{1}$. El año 1990, fue validado el Confusion Assessment Method (CAM) para el diagnóstico de delirium por médico no psiquiatra ${ }^{2}$. Este instrumento se encuentra validado para su aplicación en español, y es la herramienta más empleada en la investigación acerca del delirium. Más recientemente, fue validado el CAMICU, para diagnóstico de delirium en pacientes críticos sometidos a ventilación mecánica ${ }^{3}$. Para este instrumento, nuestro grupo desarrolló una versión validada al español en Chile ${ }^{4}$. La importancia de los métodos diagnósticos abreviados, es que dado la magnitud del fenómeno, permiten un reconocimiento rápido y simple del delirium por profesionales de la salud no psiquiatras. Por otra parte, permiten el diagnóstico del subtipo hipoactivo habitualmente no reconocido por el personal sanitario no capacitado.

El delirium alcanza una incidencia entre el 15 y $50 \%$ en adultos mayores hospitalizados por patología médica ${ }^{5,6}$. En pacientes sometidos a ventilación mecánica, su incidencia oscila entre el 60 y $85 \%{ }^{7}$. Para el DPO, la incidencia descrita oscila entre el 5 y 50\%, según sean las características demográficas, comorbilidades, el tipo de procedimiento quirúrgico, y el tipo de anestesia, entre otros. Así, las mayores incidencias de DPO descritas, se observan en pacientes sometidos a cirugía vascular, cardiocirugía y en adultos mayores de 70 años sometidos a cirugía por fractura de cadera ${ }^{8-11}$. En cirugía abdominal mayor, Koebrugge y cols, describen una incidencia de $24 \%{ }^{12}$. Patti y cols, documentan una incidencia de $18 \%$ de DPO en adultos mayores sometidos a cirugía de colon por cáncer, valor similar a lo observado por McAlpine en mujeres sometidas a cirugía por sospecha de cáncer ginecológico ${ }^{13,14}$. Cuando han sido evaluados grupos indiferenciados de adultos mayores sometidos a cirugía electiva o de urgencia, la incidencia es del 13\% en cirugía electiva, y 18\% en cirugía de urgencia.

En miras a un mejor ordenamiento de los factores de riesgo asociados a delirium postoperatorio, es recomendable emplear el esquema de factores predisponentes y precipitantes ${ }^{6}$ (Tabla 1). Los más reconocidos factores predisponentes, son la edad, el estado cognitivo, el estado funcional, el nutricional, a los que más recientemente se han incorporado factores de riesgo genéticos ${ }^{15}$.

El primer estudio que evaluó sistemáticamente la predicción del riesgo de DPO en pacientes quirúrgicos, fue desarrollado por Marcantonio en $1994^{16}$. Las variables que se asociaron a DPO fueron edad $\geq 70$ años, uso de alcohol, mal status cognitivo, mal status funcional, alteraciones preoperatorias del sodio, potasio o glicemia, cirugía torácica no cardíaca, y cirugía de aneurisma aórtico. En pacientes cardioquirúrgicos, recientemente se validó otra regla de predicción que incorpora el estado mental, la presencia de depresión, antecedentes de enfermedad cerebrovascular, y alteración en el valor de albúmina preoperatorio ${ }^{9}$. Estos instrumentos, colaboran en la estratificación de riesgo preoperatorio de DPO, sin embargo, sólo incorporan variables no modificables.

Durante los últimos años, se han registrado avances en el reconocimiento de factores de riesgo asociados al manejo perioperatorio, en los cuales potencialmente se podría intervenir para reducir el DPO. Un aspecto de interés, es el uso de fármacos 
DELIRIUM POSTOPERATORIO. UNA VENTANA HACIA UNA MEJORÍA DE LA CALIDAD Y SEGURIDAD EN LA ...

Tabla 1. Principales factores de riesgo predisponentes y precipitantes para el desarrollo de delirium postoperatorio

\begin{tabular}{|ll|}
\hline Predisponentes & Precipitantes \\
\hline Edad & Hipotensión intraoperatoria \\
\hline Deterioro cognitivo & Dolor postoperatorio \\
Dependencia funcional & Fármacos \\
Déficit visual o auditivo & Anticolinérgicos (ej: metoclopramida) \\
Desnutrición & Sedantes (ej: midazolam) \\
Polimorfismo Apo E4 & Analgésicos (ej: tramadol) \\
Abuso de alcohol & Privación de sueño \\
Cirugía torácica no cardíaca & Uso de contenciones y/o catéteres \\
Cirugía vascular & \\
\hline
\end{tabular}

que tienen el potencial de gatillar delirium. Dentro de ellos, el grupo de fármacos anticolinérgicos ha sido tradicionalmente vinculado al riesgo de delirium ${ }^{17}$. Pese a que aún hay controversia en la existencia de esta asociación ${ }^{18}$, todos los programas de prevención de delirium incorporan el uso restrictivo de estos fármacos, dentro de los cuales se encuentran varios de uso habitual como son la ranitidina, famotidina, clorfenamina, y metoclopramida entre otros. Otro grupo de fármacos asociado a delirium son las benzodiazepinas. Ello ha sido observado consistentemente tanto para DPO, como para el delirium en $\mathrm{UCI}^{19,20}$. Por lo tanto, se desaconseja su empleo salvo en usuarios previos o en síndrome de privación de alcohol.

En relación al dolor postoperatorio y al uso de opiáceos como factores de riesgo de delirium, existe a la fecha el consenso en que el dolor puede gatillar delirium ${ }^{21,22}$. Tramadol ha sido asociado de manera independiente a DPO, aunque es complejo diferenciar el impacto del dolor y/o de un analgésico particular en la aparición de delirium ${ }^{23}$. Por otra parte, el desarrollo de delirium podría limitar el uso de estrategias analgésicas que requieren de la colaboración del paciente (analgesia controlada por el paciente, $P C A$ ), aunque ello no fue documentado en un reciente estudio ${ }^{24}$.

Finalmente, la presencia de hipotensión intraoperatoria, así como la profundidad anestésica han sido sugeridos como gatillantes de $\mathrm{DPO}^{25-27}$. Faltan más estudios que confirmen estos hallazgos preliminares.

\section{Importancia del delirium postoperatorio}

A mediados de la década del 90`, y apoyados en el CAM como instrumento diagnóstico simple, rápido y reproducible, empezaron a surgir los primeros reportes que asocian la presencia de delirium con peores desenlaces sanitarios. Así es como los primeros estudios realizados en pacientes médicos revelaron una asociación independiente entre delirium y mayor estadía hospitalaria, complicaciones, necesidad de institucionalización, mortalidad alejada, status funcional, status cognitivo, y $\operatorname{costos}^{28-31}$. A partir del año 2000, y luego de la validación del CAM-ICU, empezaron los estudios de delirium en los pacientes críticos. En estos pacientes, su presencia y duración también se ha asociado de forma independiente a mortalidad, replicándose los hallazgos en relación a otros desenlaces ${ }^{32-34}$.

Para el delirium postoperatorio, los primeros reportes provienen de adultos mayores con fractura de cadera. En ellos, Marcantonio y cols, documentan deterioro funcional 1 mes post egreso hospitalario, así como mayor institucionalización y mortalidad ${ }^{35}$. Lundstrom, publica el año 2003 la presencia de asociación entre delirium y el desarrollo de demencia, en un seguimiento a 5 años. Estas observaciones, fueron posteriormente corroboradas por Bickel ${ }^{10,36}$. De la misma manera, en pacientes cardioquirúrgicos se ha observado asociación entre DPO con mortalidad, necesidad de reingreso, y menor calidad de vida ${ }^{37,38}$. En adultos mayores sometidos a cirugía general electiva, así como en aquellos sometidos a cirugía abdominal también se ha documentado mayor estadía hospitalaria y mortalidad ${ }^{12,39}$.

El gran impacto de la aparición de delirium en el desenlace de los pacientes hospitalizados, ha motivado no sólo una intensa búsqueda de los mecanismos que lo subyacen, y de mejores estrategias de prevención y terapia, sino que ha planteado la urgencia de sensibilizar a los diversos equipos médicos en este tema, de forma de implementar estrategias de prevención ${ }^{40}$. Así, el diagnóstico de delirium ha sido incorporado dentro de los indicadores de calidad de agencias internacionales como la americana Agency for Healthcare Research and Quality (www. qualitymeasures.ahrq.gov).

Uno de los aspectos no aclarados de la asociación entre delirium y peores desenlaces, son los mecanismos que llevan a que pacientes con un trastorno cerebral agudo y habitualmente reversible, tengan peor evolución al seguimiento alejado. Dentro de los factores planteados, está la pérdida de independencia funcional y cognitiva. Estos son ampliamente reconocidos como predictores de calidad de vida y mortalidad en los adultos mayores. Por otra parte, se ha sugerido que la aparición de delirium podría desenmascarar a pacientes con demencia o deterioro cognitivo leve no conocidos. Sin embargo, 
estudios que han controlado la variable estado cognitivo basal, confirman los desenlaces desfavorables del delirium.

Finalmente, en relación a los subtipos clínicos de delirium, existe aún controversia en su impacto pronóstico. Reciente información de pacientes críticos quirúrgicos, sugiere que los pacientes con subtipo hipoactivo tienen un mayor riesgo de úlceras por presión, y mortalidad en comparación con la variante mixta o hiperactiva ${ }^{41}$.

\section{Mecanismos}

Los mecanismos más estudiados en el desarrollo del delirium son:

\section{a. Deficiencia colinérgica central}

La neurotransmisión colinérgica ha sido durante muchos años relacionada con la atención y la memoria en el modelo animal. La administración de atropina en el cerebro de rata está asociada a registros electroencefalográficos similares a los observados en pacientes con delirium ${ }^{42}$. En seres humanos, la deficiencia en la transmisión colinérgica se ha descrito en la enfermedad de Alzheimer (AD) y en el deterioro cognitivo leve. Con posterioridad, se desarrolló un grupo de nuevos medicamentos: los inhibidores de la colinesterasa que han demostrado ser parcialmente eficaces para retrasar la progresión y la mejoría de algunos síntomas del Alzheimer. En delirium sin embargo, los ensayos clínicos dirigidos a mejorar la actividad colinérgica con estos fármacos, han sido negativos ${ }^{43,44}$.

\section{b. Otros neurotransmisores}

El exceso en la actividad de las vías de dopamina o glutamato, así como la actividad GABA, también se han estudiado en delirium. La vía GABAérgica está bien documentada, en particular por la asociación entre uso de benzodiazepinas y delirium. Investigación en pacientes en ventilación mecánica, apunta al impacto favorable de la reducción de benzodiazepinas, y su reemplazo por alfa-2 agonistas como sedante de elección ${ }^{45}$. Para otros neurotransmisores, la información disponible hasta la fecha es objeto de controversia.

\section{c. Inflamación y delirium}

Un incremento en mediadores inflamatorios como IL-6 e IL-8 ha sido observado en pacientes con delirium, en comparación con controles ${ }^{46}$. Un aumento de quemokinas ha sido observado en pacientes sometidos a cardiocirugía que desarrollan delirium. En pacientes quirúrgicos, se ha documentado asociación entre la curva de proteína $C$ reactiva y la evolución temporal del delirium ${ }^{47}$. A pesar de estos resultados positivos, y de investigación básica de la relación inflamación-delirium, hay limitaciones teóricas y en la aplicación de la información disponible ${ }^{48}$. Los estudios han sido habitualmente de caso y control, en pacientes que ya han desarrollado delirium. Ello no permite evaluar asociación temporal. Por otro lado, estrategias de modulación de la respuesta inflamatoria, no han documentado impacto en la incidencia de delirium.

\section{d. Ambiente no farmacológico}

La evidencia del impacto de la prevención no farmacológica en la incidencia de delirium, sugiere que en el cerebro frágil la preservación de ciertas rutinas, la estimulación cognitiva y motora temprana, y la reducción de los estímulos nociceptivos, son capaces de reducir el impacto del delirium. Los mecanismos por los que ello tiene un impacto positivo, son desconocidos.

\section{e. Hipoperfusión cerebral perioperatoria}

La hipotensión intraoperatoria, ha sido descrita como factor de riesgo de delirium ${ }^{13}$. Es plausible, aunque no hay información sólida a la fecha, que en cerebros anatómica y funcionalmente vulnerables, pequeños cambios hemodinámicos, de la perfusión y oxigenación cerebral durante la cirugía, y en el período postoperatorio puedan asociarse a delirium. En la actualidad, nuestro grupo desarrolla el proyecto Fondecyt de Iniciación 11100246 que pretende explorar esta hipótesis en adultos mayores sometidos a cirugía de colon electiva, a través del monitoreo no invasivo de la oxigenación cerebral (empleando para ello brain near-infrared spectroscopy, NIRS cerebral), neuroimágenes, y evaluación seriada de la perfusión global con Lactato y Saturación venosa central continua de oxígeno $\left(\mathrm{S}_{\mathrm{cv}} \mathrm{O}_{2}\right)$.

En resumen, a pesar de que hay avances en el conocimiento fisiopatológico, aún existen grandes brechas en el conocimiento de este complejo fenómeno cerebral. Un mejor conocimiento de los mecanismos subyacente, puede conducir al desarrollo de terapias más eficaces que las actualmente disponibles.

\section{Prevención}

Dado el gran impacto sanitario del delirium, a finales de la década de los $90^{\circ}$, surgieron los primeros estudios evaluando diferentes estrategias de prevención. El primero que documentó una reducción del delirium, fue desarrollado por Inouye y colaboradores $^{49}$. Este fue un estudio desarrollado en mayores de 70 años admitidos a salas de medicina interna, con un diseño quasiexperimental. Los pacientes admitidos a las unidades intervenidas, recibieron el Elder Life Program, que consiste en un equipo mul- 
tidisciplinario (geriatras, enfermeras, kinesiólogos, y voluntarios entrenados) que realizaban actividades en seis dominios mediante protocolos predefinidos. Estos eran el estado cognitivo, evitar la privación de sueño, reducir la inmovilización, limitar los déficits sensoriales visual y auditivo, y evitar la deshidratación. La incidencia de delirium se redujo desde $15 \%$ a $9,9 \%(p=0,02)$. El primer estudio en pacientes quirúrgicos, fue efectuado en mayores de 65 años con fractura de cadera. En este estudio, el grupo intervención fue sometido a un sistema de interconsulta geriátrica proactiva desde el preoperatorio. Luego se realizaba visita diaria, y recomendaciones basadas en un protocolo estructurado. Este estudio documentó una reducción en la incidencia de delirium desde $50 \%$ a $32 \%$, además de una menor incidencia de delirium severo ${ }^{50}$. Estos hallazgos, han sido posteriormente reproducidos en otras poblaciones de pacientes, aunque existen pocos estudios en grupos heterogéneos de pacientes quirúrgicos ${ }^{51,52}$. En pacientes críticos, tampoco hay estudios de estrategias no farmacológicas, aunque ensayos de terapia física y ocupacional precoz cuyo desenlace primario es independencia funcional, han encontrado una reducción significativa en la duración de delirium ${ }^{53}$. En base a los hallazgos encontrados, existe un buen nivel de evidencia respecto del rol de estrategias de prevención no farmacológica de delirium en adultos mayores hospitalizados. Pese a estar consistentemente recomendadas, su implementación ha sido muy escasa a la fecha. En nuestro centro, se realiza sistemáticamente en la unidad geriátrica de agudos y en las unidades de intermedio de la Unidad de Pacientes Críticos.

Respecto a prevención farmacológica de delirium, la evidencia ha sido discordante. Los estudios con procolinérgicos han sido negativos. Estudios con haloperidol, sugieren un rol de éste en reducir la duración y severidad del delirium en pacientes sometidos a cirugía de cadera, y más recientemente en reducir la incidencia de delirium en pacientes críticos quirúrgicos ${ }^{54,55}$. Pequeños estudios con risperidona y olanzapina sugieren también un rol para estos fármacos ${ }^{56,57}$. Aunque los recientes estudios de prevención farmacológica con neurolépticos han sido positivos, aún no está claro si estos beneficios son aplicables a otras poblaciones de pacientes en riesgo. Por ello, y por los riesgos potenciales de este grupo de fármacos, a la fecha el uso de neurolépticos en profilaxis es inusual.

\section{Tratamiento}

Ante la aparición de delirium postoperatorio, es necesario llevar a cabo un enfrentamiento estructurado, de manera de minimizar las omisiones, y reducir los efectos adversos asociados a la terapia farmacológica (Tabla 2).

Lo primero, es realizar una evaluación de la presencia de factores gatillantes. El objetivo de esta evaluación es descartar que el delirium sea una manifestación precoz de alguna complicación médica o quirúrgica postoperatoria. Es por ello, que aunque exista un paciente de elevado riesgo basado en la estratificación de riesgo preoperatorio, es necesario realizar una evaluación clínica y de laboratorio bási-

\section{Tabla 2. Enfrentamiento sistemático del paciente quirúrgico con delirium postoperatorio, Unidad de Pacientes Críticos Hospital Clínico Universidad de Chile}

\section{Evaluar factores precipitantes}

- Examen clínico para evaluar complicaciones médicas y/o quirúrgicas

- Laboratorio complementario básico (Hemograma, PCR, función renal y hepática, electrolitos, ECG y marcadores de daño miocárdico)

- Inicio de terapia si se documenta nuevo factor gatillante

- En caso de focalidad al examen neurológico, o forma de presentación inhabitual de delirium, evaluación por neurología y considerar exámenes adicionales para descarte de patología cerebral primaria

\section{Enfrentamiento no farmacológico}

- Evitar fármacos precipitantes

- Corregir déficit sensoriales

- Reforzar educación a personal sanitario

- Educar a familia

- Propiciar movilización precoz

- Reorientación y estimulación cognitiva

- Si delirium hiperactivo de difícil control, evaluar necesidad de mayor monitorización y terapia en Unidad de Pacientes Críticos

\section{Terapia farmacológica}

- Considerar en pacientes con delirium mixto o hiperactivo

- Evaluar intervalo QT, y presencia de condiciones neurológicas que contraindiquen empleo de neurolépticos (Ej: enfermedad de Parkinson)

- Inicio de neurolépticos: Haloperidol 0,5-1 mg vía oral cada 8 horas; Risperidona (0,5-1 mg cada 8 horas; o Quetiapina 25 a $100 \mathrm{mg}$ diarios). En general privilegiar mayores dosis entre 21:00 y 23:00 horas si predominio de síntomas positivos nocturnos

- Evitar dosis elevadas de neurolépticos y combinaciones con sedantes en pacientes con agitación severa

\section{Reevaluación diaria}

- Evaluar presencia y severidad del delirium 2 veces al día

- Evolución del (los) precipitante (s) en caso de documentarse

- Evaluar intervalo QT y signos extrapiramidales

- Ajuste diario de dosis de neurolépticos, según eficacia terapéutica y presencia de efectos adversos 
ca, que incluya oxigenación, glicemia, función renal, medio interno, respuesta inflamatoria, y marcadores de daño miocárdico, que permitan excluir la presencia de una complicación médica que se manifiesta inicialmente como delirium (ej: infarto al miocardio postoperatorio). Asimismo, es muy relevante la evaluación del equipo quirúrgico tratante, en particular si existe un mayor riesgo de que pudiera aparecer una complicación quirúrgica. En este escenario, es de particular relevancia fomentar la interacción entre el equipo quirúrgico, y el equipo de medicina interna y/o medicina intensiva que colabora en la atención de estos pacientes. Inusualmente, y ante la presencia de alteraciones focales al examen neurológico, o una forma de presentación inhabitual de este cuadro (por su severidad, momento de presentación, o duración), es necesario recurrir a evaluación formal por neurología, neuroimágenes, electroencefalograma, u otro examen complementario con la finalidad de descartar alteraciones orgánicas u otro diagnóstico diferencial. En relación al manejo no farmacológico de un paciente que desarrolla delirium, especial atención debemos tener en evitar los efectos adversos asociados al exceso de fármacos sedantes y/o neurolépticos. Es clave favorecer una adecuada monitorización, y la seguridad de pacientes con severas manifestaciones hiperactivas, de forma de evitar daños o retiros de dispositivos médicos como sondas o drenajes. Por ello, en general, no es susceptible su manejo en sala común, debiendo ser trasladados a unidades que permitan mayor monitoreo y vigilancia.

El rol de estrategias multimodales de prevención, y su impacto en la terapia del delirium ya constituido, no cuentan con evidencia sólida que lo respalde, sin embargo, son recomendadas en las guías disponibles $^{58,59}$.

Finalmente, el rol de los neurolépticos en el manejo del paciente con delirium es colaborar en el control de los síntomas positivos de pacientes con formas mixtas o hiperactivas. A la fecha no existe un elevado nivel de evidencia en este ámbito, y se carece de estudios de elevada calidad y tamaño muestral que documenten fehacientemente una superioridad de los neurolépticos en relación al placebo. El más empleado, y recomendado es el Haloperidol $^{60}$. Se recomiendan dosis bajas vía oral, partiendo con 0,25 a 0,5 mg, hasta 3 a $5 \mathrm{mg}$ diarios. En los estudios realizados, se emplea entre 5-7 días titulando diariamente la dosis según sea la severidad de los síntomas. Haloperidol, tiene formulación endovenosa, aunque por lo general se desaconseja su empleo por un mayor riesgo de arritmias, que motivaron una alerta de FDA ${ }^{61}$. Los efectos adversos de los neurolépticos como grupo, y en particular de Haloperidol son la prolongación del intervalo QT, con el riesgo de Torsión de Puntas. Dentro de los efectos adversos neurológicos, el más temido es el síndrome neuroléptico maligno, de muy baja frecuencia. La complicación más frecuente del uso de haloperidol son los trastornos extrapiramidales, que pueden desarrollarse hasta en el 10-15\% de adultos mayores que reciben Haloperidol en dosis superior a $3 \mathrm{mg} /$ día.

Durante los últimos 10 años, y motivados por la controversial eficacia y los problemas de seguridad con haloperidol, han surgido estudios que evalúan el rol de los neurolépticos atípicos en delirium. Así es como hay estudios con Risperidona ${ }^{62}$, Quetiapina ${ }^{63,64}$, y Olanzapina ${ }^{65,66}$. Aunque la evidencia aún es insuficiente, existen progresivos reportes de similar o superior eficacia a haloperidol, con menores efectos adversos. Localmente, y en virtud de un trabajo conjunto entre el equipo de UPC y psiquiatría, la mayor parte de los pacientes hospitalizados que requieren terapia farmacológica del delirium, lo hacen con quetiapina en dosis bajas (12,5 a $100 \mathrm{mg} /$ día). Ocasionalmente, en pacientes críticos jóvenes las dosis pueden llegar a 400-600 mg diarios.

En suma, el manejo farmacológico del delirium sigue siendo un campo abierto a la investigación clínica, y donde debe sopesarse el riesgo-beneficio de las opciones disponibles, evitando el exceso de sedantes, y sus riesgo asociados. No olvidar el rol central del manejo no farmacológico, aunque su evidencia en terapia es insuficiente.

\section{Conclusiones}

El delirium postoperatorio es una complicación médica relevante y frecuente en el adulto mayor quirúrgico. El oportuno diagnóstico, y la implementación de estrategias de prevención no farmacológicas son un desafío para la organización de los servicios quirúrgicos. Una vez diagnosticado, un enfrentamiento organizado que propicie la seguridad del paciente es clave en su pronta resolución.

\section{Agradecimientos}

Los autores agradecen a Conicyt, el cual a través del proyecto Fondecyt de Iniciación 11100246, da su apoyo y financiamiento para el desarrollo de investigación en delirium postoperatorio.

\section{Referencias}

1. Tucker GJ. The diagnosis of delirium and DSM-IV. Dement Geriatr Cogn Disord. 1999;10:359-63.

2. Inouye SK, van Dyck CH, Alessi CA, Balkin S, Siegal 
AP, Horwitz RI. Clarifying confusion: the confusion assessment method. A new method for detection of delirium. Ann Intern Med. 1990;113:941-8.

3. Ely EW, Inouye SK, Bernard GR, Gordon S, Francis J, May L, et al. Delirium in mechanically ventilated patients: validity and reliability of the confusion assessment method for the intensive care unit (CAMICU). JAMA 2001;286:2703-10.

4. Tobar E, Romero C, Galleguillos T, Fuentes P, Cornejo R, Lira MT, et al. [Confusion Assessment Method for diagnosing delirium in ICU patients (CAM-ICU): cultural adaptation and validation of the Spanish version]. Med Intensiva 2010;34:4-13.

5. Inouye SK, Viscoli CM, Horwitz RI, Hurst LD, Tinetti ME. A predictive model for delirium in hospitalized elderly medical patients based on admission characteristics. Ann Intern Med. 1993;119:474-81.

6. Inouye SK. Delirium in older persons. N Engl J Med. 2006;354:1157-65.

7. Ely EW, Stephens RK, Jackson JC, Thomason JW, Truman B, Gordon S, et al. Current opinions regarding the importance, diagnosis, and management of delirium in the intensive care unit: a survey of 912 healthcare professionals. Crit Care Med. 2004;32:106-12.

8. Rudolph JL, Jones RN, Rasmussen LS, Silverstein JH, Inouye SK, Marcantonio ER. Independent vascular and cognitive risk factors for postoperative delirium. Am J Med. 2007;120:807-13.

9. Rudolph JL, Jones RN, Levkoff SE, Rockett C, Inouye SK, Sellke FW, et al. Derivation and validation of a preoperative prediction rule for delirium after cardiac surgery. Circulation. 2009;119:229-36.

10. Lundstrom M, Edlund A, Bucht G, Karlsson S, Gustafson Y. Dementia after delirium in patients with femoral neck fractures. J Am Geriatr Soc. 2003;51:1002-6.

11. Bruce AJ, Ritchie CW, Blizard R, Lai R, Raven P. The incidence of delirium associated with orthopedic surgery: a meta-analytic review. Int Psychogeriatr. 2007;19:197-214.

12. Koebrugge B, Koek HL, van Wensen RJ, Dautzenberg PL, Bosscha K. Delirium after abdominal surgery at a surgical ward with a high standard of delirium care: incidence, risk factors and outcomes. Dig Surg. 2009;26:63-8.

13. Patti R, Saitta M, Cusumano G, Termine G, Di Vita G. Risk factors for postoperative delirium after colorectal surgery for carcinoma. Eur J Oncol Nurs. 2011;15:51923.

14. McAlpine JN, Hodgson EJ, Abramowitz S, Richman SM, Su Y, Kelly MG, et al. The incidence and risk factors associated with postoperative delirium in geriatric patients undergoing surgery for suspected gynecologic malignancies. Gynecol Oncol. 2008;109:296-302.

15. Leung JM, Sands LP, Wang Y, Poon A, Kwok PY, Kane JP, et al. Apolipoprotein E e4 allele increases the risk of early postoperative delirium in older pa- tients undergoing noncardiac surgery. Anesthesiology 2007; 107:406-11.

16. Marcantonio ER, Goldman L, Mangione CM, Ludwig LE, Muraca B, Haslauer CM, et al. A clinical prediction rule for delirium after elective noncardiac surgery. JAMA. 1994;271:134-9.

17. Alagiakrishnan $\mathrm{K}$, Wiens $\mathrm{CA}$. An approach to drug induced delirium in the elderly. Postgrad Med J. 2004;80:388-93.

18. Campbell N, Perkins A, Hui S, Khan B, Boustani M. Association between prescribing of anticholinergic medications and incident delirium: a cohort study. J Am Geriatr Soc. 2011;59 Suppl 2:S277-81.

19. Marcantonio ER, Juarez G, Goldman L, Mangione CM, Ludwig LE, Lind L, et al. The relationship of postoperative delirium with psychoactive medications. JAMA 1994;272:1518-22.

20. Pandharipande P, Shintani A, Peterson J, Pun BT, Wilkinson GR, Dittus RS, et al. Lorazepam is an independent risk factor for transitioning to delirium in intensive care unit patients. Anesthesiology 2006;104:21-6.

21. Lynch EP, Lazor MA, Gellis JE, Orav J, Goldman L, Marcantonio ER. The impact of postoperative pain on the development of postoperative delirium. Anesth Analg. 1998;86:781-5.

22. Vaurio LE, Sands LP, Wang Y, Mullen EA, Leung JM. Postoperative delirium: the importance of pain and pain management. Anesth Analg. 2006;102:1267-73.

23. Brouquet A, Cudennec T, Benoist S, Moulias S, Beauchet A, Penna C, et al. Impaired mobility, ASA status and administration of tramadol are risk factors for postoperative delirium in patients aged 75 years or more after major abdominal surgery. Ann Surg. 2010;251:759-65.

24. Leung JM, Sands LP, Paul S, Joseph T, Kinjo S, Tsai $\mathrm{T}$. Does postoperative delirium limit the use of patientcontrolled analgesia in older surgical patients? Anesthesiology 2009;111:625-31.

25. Marcantonio ER, Goldman L, Orav EJ, Cook EF, Lee $\mathrm{TH}$. The association of intraoperative factors with the development of postoperative delirium. Am J Med. 1998;105:380-4.

26. Sieber FE, Zakriya KJ, Gottschalk A, Blute MR, Lee $\mathrm{HB}$, Rosenberg PB, et al. Sedation depth during spinal anesthesia and the development of postoperative delirium in elderly patients undergoing hip fracture repair. Mayo Clin Proc. 2010;85:18-26.

27. Tognoni P, Simonato A, Robutti N, Pisani M, Cataldi A, Monacelli F, et al. Preoperative risk factors for postoperative delirium (POD) after urological surgery in the elderly. Arch Gerontol Geriatr. 2011;52:e166-9.

28. Inouye SK, Rushing JT, Foreman MD, Palmer RM, Pompei P. Does delirium contribute to poor hospital outcomes? A three-site epidemiologic study. J Gen Intern Med. 1998;13:234-42.

29. Leslie DL, Zhang Y, Holford TR, Bogardus ST, Leo- 
Summers LS, Inouye SK. Premature death associated with delirium at 1-year follow-up. Arch Intern Med. 2005;165:1657-62.

30. Leslie DL, Marcantonio ER, Zhang Y, Leo-Summers L, Inouye SK. One-year health care costs associated with delirium in the elderly population. Arch Intern Med. 2008;168:27-32.

31. Witlox J, Eurelings LS, de Jonghe JF, Kalisvaart KJ, Eikelenboom P, van Gool WA. Delirium in elderly patients and the risk of postdischarge mortality, institutionalization, and dementia: a meta-analysis. JAMA 2010;304:443-51.

32. Ely EW, Shintani A, Truman B, Speroff T, Gordon SM, Harrell FE, Jr., et al. Delirium as a predictor of mortality in mechanically ventilated patients in the intensive care unit. JAMA 2004;291:1753-62.

33. Pisani MA, Kong SY, Kasl SV, Murphy TE, Araujo KL, Van Ness PH. Days of delirium are associated with 1-year mortality in an older intensive care unit population. Am J Respir Crit Care Med. 2009;180:1092-7.

34. Ouimet S, Kavanagh BP, Gottfried SB, Skrobik Y. Incidence, risk factors and consequences of ICU delirium. Intensive Care Med. 2007;33:66-73.

35. Marcantonio ER, Flacker JM, Michaels M, Resnick NM. Delirium is independently associated with poor functional recovery after hip fracture. J Am Geriatr Soc. 2000;48:618-24.

36. Bickel H, Gradinger R, Kochs E, Forstl H. High risk of cognitive and functional decline after postoperative delirium. A three-year prospective study. Dement Geriatr Cogn Disord. 2008;26:26-31.

37. Koster S, Hensens AG, van der Palen J. The long-term cognitive and functional outcomes of postoperative delirium after cardiac surgery. Ann Thorac Surg. 2009;87:1469-74.

38. Koster S, Hensens AG, Schuurmans MJ, van der Palen J. Consequences of Delirium After Cardiac Operations. Ann Thorac Surg. 2011. [Epub ahead of print].

39. Ansaloni L, Catena F, Chattat R, Fortuna D, Franceschi C, Mascitti P, et al. Risk factors and incidence of postoperative delirium in elderly patients after elective and emergency surgery. Br J Surg. 2010;97:273-80.

40. Inouye SK, Schlesinger MJ, Lydon TJ. Delirium: a symptom of how hospital care is failing older persons and a window to improve quality of hospital care. Am J Med. 1999;106:565-73.

41. Robinson TN, Raeburn CD, Tran ZV, Brenner LA, Moss M. Motor subtypes of postoperative delirium in older adults. Arch Surg. 2011;146:295-300.

42. Trzepacz PT, Leavitt M, Ciongoli K. An animal model for delirium. Psychosomatics. 1992;33:404-15.

43. Marcantonio ER, Palihnich K, Appleton P, Davis RB. Pilot randomized trial of donepezil hydrochloride for delirium after hip fracture. J Am Geriatr Soc. 2011;59 Suppl 2:S282-8.

44. Gamberini M, Bolliger D, Lurati Buse GA, Burkhart
CS, Grapow M, Gagneux A, et al. Rivastigmine for the prevention of postoperative delirium in elderly patients undergoing elective cardiac surgery-a randomized controlled trial. Crit Care Med. 2009;37:1762-8.

45. Riker RR, Shehabi Y, Bokesch PM, Ceraso D, Wisemandle W, Koura F, et al. Dexmedetomidine vs midazolam for sedation of critically ill patients: a randomized trial. JAMA 2009;301:489-99.

46. de Rooij SE, van Munster BC, Korevaar JC, Levi M. Cytokines and acute phase response in delirium. J Psychosom Res. 2007;62:521-5.

47. Macdonald A, Adamis D, Treloar A, Martin F. Creactive protein levels predict the incidence of delirium and recovery from it. Age Ageing. 2007;36:222-5.

48. van Gool WA, van de Beek D, Eikelenboom P. Systemic infection and delirium: when cytokines and acetylcholine collide. Lancet 2010;375:773-5.

49. Inouye SK, Bogardus ST, Jr., Charpentier PA, Leo-Summers L, Acampora D, Holford TR, et al. A multicomponent intervention to prevent delirium in hospitalized older patients. N Engl J Med. 1999;340:669-76.

50. Marcantonio ER, Flacker JM, Wright RJ, Resnick NM. Reducing delirium after hip fracture: a randomized trial. J Am Geriatr Soc. 2001;49:516-22.

51. Lundstrom M, Edlund A, Karlsson S, Brannstrom B, Bucht G, Gustafson Y. A multifactorial intervention program reduces the duration of delirium, length of hospitalization, and mortality in delirious patients. J Am Geriatr Soc. 2005;53:622-8.

52. Naughton BJ, Saltzman S, Ramadan F, Chadha N, Priore R, Mylotte JM. A multifactorial intervention to reduce prevalence of delirium and shorten hospital length of stay. J Am Geriatr Soc. 2005;53:18-23.

53. Schweickert WD, Pohlman MC, Pohlman AS, Nigos C, Pawlik AJ, Esbrook CL, et al. Early physical and occupational therapy in mechanically ventilated, critically ill patients: a randomised controlled trial. Lancet. 2009;373:1874-82.

54. Kalisvaart KJ, de Jonghe JF, Bogaards MJ, Vreeswijk R, Egberts TC, Burger BJ, et al. Haloperidol prophylaxis for elderly hip-surgery patients at risk for delirium: a randomized placebo-controlled study. J Am Geriatr Soc. 2005;53:1658-66.

55. Wang W, Li HL, Wang DX, Zhu X, Li SL, Yao GQ, et al. Haloperidol prophylaxis decreases delirium incidence in elderly patients after noncardiac surgery: A randomized controlled trial. Crit Care Med. 2011. [Epub ahead of print].

56. Larsen KA, Kelly SE, Stern TA, Bode RH, Jr., Price LL, Hunter DJ, et al. Administration of olanzapine to prevent postoperative delirium in elderly joint-replacement patients: a randomized, controlled trial. Psychosomatics 2010;51:409-18.

57. Prakanrattana U, Prapaitrakool S. Efficacy of risperidone for prevention of postoperative delirium in cardiac surgery. Anaesth Intensive Care 2007;35:714-9. 
58. Tropea J, Slee JA, Brand CA, Gray L, Snell T. Clinical practice guidelines for the management of delirium in older people in Australia. Australas J Ageing 2008;27:150-6.

59. Tahir TA, Morgan E, Eeles E. NICE guideline: evidence for pharmacological treatment of delirium. J Psychosom Res. 2011;70:197-8.

60. Practice guideline for the treatment of patients with delirium. American Psychiatric Association. Am J Psychiatry. 1999;156(5 Suppl):1-20.

61. Meyer-Massetti C, Cheng CM, Sharpe BA, Meier CR, Guglielmo BJ. The FDA extended warning for intravenous haloperidol and torsades de pointes: how should institutions respond? J Hosp Med. 2010;5:E8-16.

62. Han CS, Kim YK. A double-blind trial of risperidone and haloperidol for the treatment of delirium. Psycho- somatics 2004;45:297-301.

63. Devlin JW, Roberts RJ, Fong JJ, Skrobik Y, Riker RR, Hill NS, et al. Efficacy and safety of quetiapine in critically ill patients with delirium: a prospective, multicenter, randomized, double-blind, placebo-controlled pilot study. Crit Care Med. 2010;38:419-27.

64. Tahir TA, Eeles E, Karapareddy V, Muthuvelu P, Chapple S, Phillips B, et al. A randomized controlled trial of quetiapine versus placebo in the treatment of delirium. $\mathrm{J}$ Psychosom Res. 2010;69:485-90.

65. Skrobik YK, Bergeron N, Dumont M, Gottfried SB. Olanzapine vs haloperidol: treating delirium in a critical care setting. Intensive Care Med. 2004;30:444-9.

66. Kim SW, Yoo JA, Lee SY, Kim SY, Bae KY, Yang SJ, et al. Risperidone versus olanzapine for the treatment of delirium. Hum Psychopharmacol. 2010;25:298-302. 